\title{
Peculiarities of solid particles separation in an unsteady turbulent flow of a pneumatic centrifugal classifier
}

\author{
Pavel Zyatikov a and Alecsander Roslyak \\ National Research Tomsk State University, Lenin Avenue, 36, 634050 Tomsk, Russia
}

\begin{abstract}
The task of developing a centrifugal air classifier is to improve the efficiency of classification and to extend the range of regulation for separation boundaries of various micropowders, especially in the field of particle sizes equal to less than 10 microns.
\end{abstract}

The mechanism of centrifugal separation of particles is considered in [1], where it is shown that motion of particles in a separation zone is both of a deterministic and random character. As currently it appears impossible to create an accurate model of the process taking into account the polydispersity of a powdered material, the concentration of a heterogeneous medium, the rotation of particles, as well as their interactions with each other and the channel walls, the turbulent fluctuations and the inverse effect of particles onto the carrier flow, it is advisable to consider the aerodynamics of the carrier flow and motions of individual particles in the carrier stream with a known velocity distribution separately [2].

Let us consider the physical formulation of the problem for a profiled zone of a separation classifier (Fig. 1). The geometry of the separation classifier's zone, which actually is the zone in which the process of separating a powder into large and small products takes place, consists of two discs spaced $\mathrm{H}$ from each other, which rotate at a certain angular velocity $\Omega_{\mathrm{d}}$ around the axis OZ.

The bottom disc is flat, the upper one is profiled varying as $Z=f(R)$. Along its perimeter the structure receives an outside air flow (cross section $A-A$ ) with a certain angular velocity $\Omega_{\mathrm{g}}$ and a radial velocity component $U_{1}$. Then, due to pressure difference, it passes through the working area of the structure and leaves it through the cross section $C-C$. Through the lower outlet (cross section $R_{2}-R_{3}$ ) an additional gas flow $Q_{\text {add }}$ is supplied at an angular velocity $\Omega_{\text {add }}$ together with particles which, under the effect of the centrifugal and the aerodynamic forces, fall into coarse and fine fractions. Moreover, due to prevalence of the aerodynamic force, the fine separation product passes through the working area and it is removed from the separation element in the cross section $C-C$. Under the action of the centrifugal force the coarse fraction extraction is conducted through the cross section $A-A$.

Formation of areas with a high concentration of particles not only degrades the aerodynamic situation in the separation zone, but also prevents the passage of fine particles into the fine separation product. Using unsteady oscillations of the carrier medium rate allows reduction of residence time for particles of a boundary size and a size close to it and, thereby, reduction of rope-formed compositions of particles in the separation zone of the centrifugal machine.

\footnotetext{
${ }^{a}$ Corresponding author: zpavel@niipmm.tsu.ru
}

This is an Open Access article distributed under the terms of the Creative Commons Attribution License 4.0, which permits unrestricted use, distribution, and reproduction in any medium, provided the original work is properly cited. 


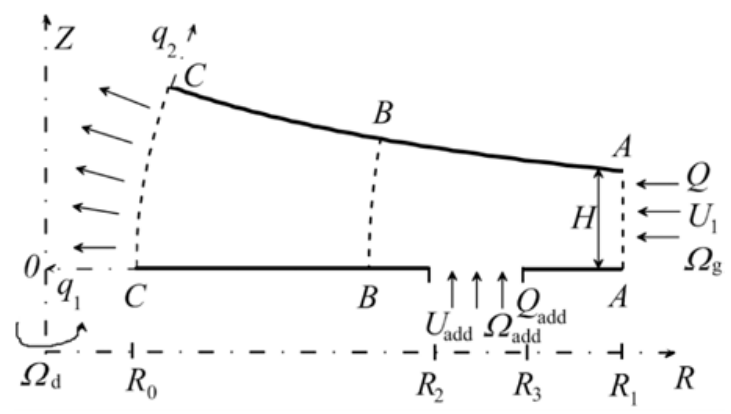

Figure 1. Scheme of the separation element of a pneumatic centrifugal classifier with a profiled top disc.

The physical essence of the classification consists in the fact that while separating fine powders in a swirled dust and gas flow directed towards the centre of rotation, the particle with a diameter $\delta$ is subject to the force of aerodynamic drag in accordance with Stokes' law [1]

$$
F_{c}=3 \pi \cdot \mu \cdot \delta \cdot\left|U_{r}-V_{r}\right|,
$$

where $\mu$ is the coefficient of dynamic gas viscosity and $U_{r}, V_{r}$ are the radial velocity components of the gas and the particles respectively.

On the other hand, due to its rotation in the separation zone with a circumferential velocity component $V_{\varphi}$, the particle is under the action of the centrifugal force

$$
F_{n}=\frac{1}{6} \cdot \pi \cdot \delta^{3} \cdot \rho_{m} \cdot \frac{V_{\varphi}^{2}}{R},
$$

where $\rho_{m}$ - the density of the particle material and $R$ is the radius around which the particle rotates.

In case of the centrifugal and the aerodynamic forces being equal, particles of a boundary size move along equilibrium paths and for them $V_{r}=0$. For fine powder particles the circumferential velocity component coincides with that of gas. Equating the values of the aerodynamic (1) and the centrifugal (2) forces and performing relevant transformations for boundary sized particles we obtain

$$
\delta_{s p}^{2}=\frac{U_{r}}{U_{\varphi}^{2}} \cdot R \cdot \frac{18 \mu}{\rho_{m}} .
$$

The value $\tau=\rho_{m} \delta^{2} / 18 \eta$ is the time of particles dynamic relaxation characterizing the rate of dynamic properties change for particles with diameters $\delta$, density $\rho_{m}$ and kinematic viscosity $v$, as $\eta=\rho v$. It is determined [1] that particle velocity is dependent on gas velocity and, in addition, on the parameter $\tau$. If the air flow rate is variable, depending on how fast it changes the degree of particle entertainment by a gas stream will depend on the dynamic relaxation time.

The present paper deals with the method and organization [3] of centrifugal classification of particles in a pulsating flow. In the separation zone of the apparatus a pulsating air flow is created, with the pulsation period being equal to the time of dynamic relaxation for particles of a boundary size. A powder is supplied into the classification zone where it undergoes the effect of opposing forces of aerodynamic drag and mass. The powder particles which are larger (smaller) than boundary ones, under the influence of the prevailing mass (aerodynamic constant component) force, are classified. Particles of boundary and near-boundary sizes, in turn, being subject to the effect of the pulsating component of the aerodynamic force, show unsteady oscillatory motion which exhibits their individual velocity properties leading to their multidirectional deterministic movement.

Figure 2. shows an apparatus for implementation of this method. A pneumatic centrifugal classifier consists of a body 1 , a collector 2 with nozzles 3 , a rotor 4 with a drive 5 and disc elements forming the 


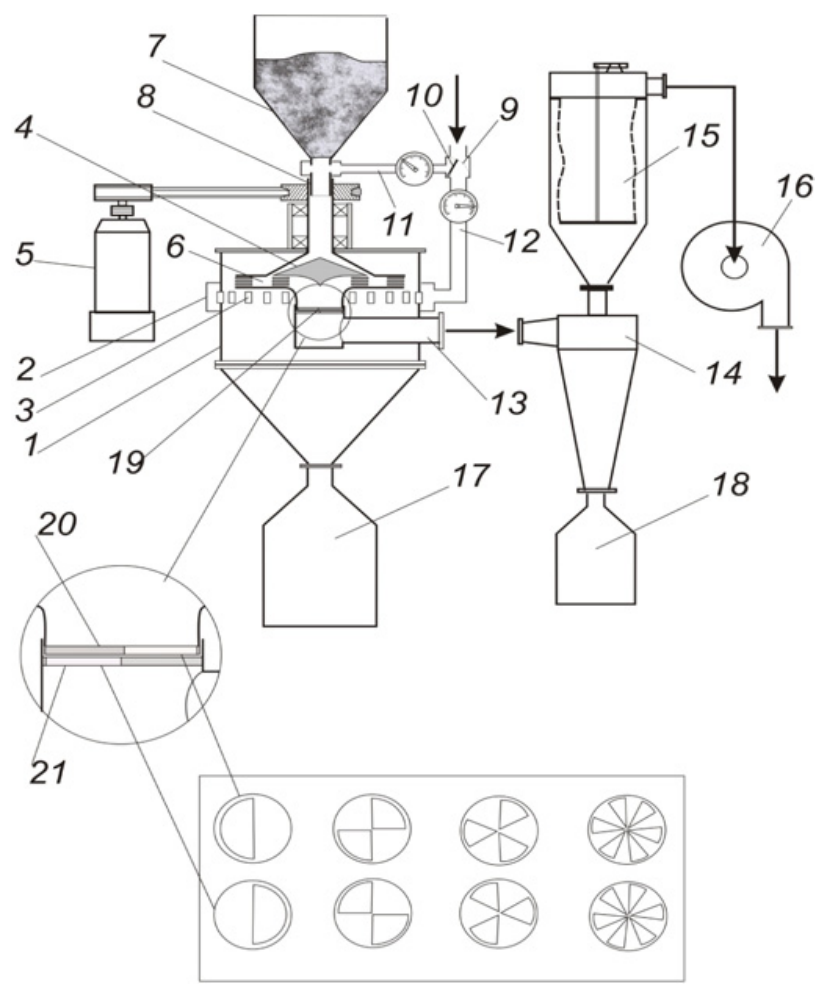

Figure 2. Pneumatic centrifugal classifier with a pulsating gas flow.

separation zone 6 , a meter 7 with a neck 8 , an air distribution device 9 with a flap 10, the first discharge connection and a flow measuring unit 11 , the second discharge connection and a flow measuring unit 12. The classifier's delivery outlet 13 is connected to a cyclone 14 , a filter 15 , a high pressure fan 16 , coarse fraction receiving hoppers 17 and those for the fine fraction 18.

The pulsator 19 consisting of two coaxial discs with windows, one of which 20 is fixed to a rotating rotor and the other 21 - to a stationary outlet conduit of the classifier 13, provides a radial component velocity pulsation of the carrier stream as it leaves the fine fraction separation zone. Wheels 20, 21 of the pulsator 19 are replaceable and they are made with a different number of windows for the purpose of changing pulsation frequency from 10 to 5000 1/s. The pulsating aerodynamic force produces disaggregation of the fine fraction, due to the difference in relaxation time for particles of different sizes, which provides final destruction of agglomerates.

Experimental research of particles classification process in a pulsating flow was carried out basing on the following materials: emulsion polyvinylchloride PVC $\left(\rho \mathrm{m}=1400 \mathrm{~kg} / \mathrm{m}^{3}\right)$, quartz sand $\mathrm{SiO}_{2}$ $\left(\rho \mathrm{m}=2650 \mathrm{~kg} / \mathrm{m}^{3}\right)$, titanium carbide powder TiC $\left(\rho \mathrm{m}=4920 \mathrm{~kg} / \mathrm{m}^{3}\right.$ with separation boundary of 10 microns, 50 microns, 63 microns, 100 microns). Moreover, the variation range of an air flow pulsation period $\mathrm{T}$ for each case was chosen so that the value of dynamic relaxation time for boundary sized particles $\tau$ was within the variation range of pulsation period T. For emulsion PVC with separation boundary of 63 microns the dynamic relaxation $\tau$ is equal to $0.0171 \mathrm{p}$.

A boundary size of 5 microns in a classifier with a separation zone radius of $0.12 \mathrm{~m}$ and a carrier flow rate of $0.05 \mathrm{~m}^{3} / \mathrm{s}$ is achieved at the rotor speed $\omega=60 \mathrm{rev} / \mathrm{s}$. For achieving the required pulsation frequency in a given mode of separation it is necessary to realize $n=f / \omega \approx 80$ windows in the pulsator discs. 


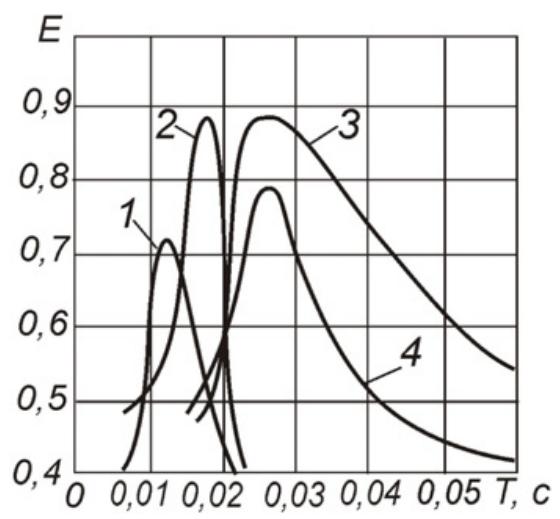

Figure 3. Dependence of the efficiency of classification on the period of an air flow pulsation: $1-$ PVC (s.b. $\delta=$ $50 \mathrm{~mm}), 2-$ PVC (s.b. $\delta=63 \mathrm{~mm}), 3$ - titanium carbide (s.b. $\delta=63 \mathrm{~mm}), 4-$ quartz sand $($ s.b. $\delta=50 \mathrm{~mm})$.

For a boundary size of 10 microns, under the same conditions and the rotor speed $\omega=41 \mathrm{rev} / \mathrm{s}$, it is necessary to realize 30 windows in the pulsator discs.

For a boundary size of 20 microns, under the same conditions and the rotor speed $\omega=41 \mathrm{rev} / \mathrm{s}$, it is necessary to realize 13 windows in the pulsator discs.

Thus, when creating a pulsating flow with a pulsation period equal to dynamic relaxation time for boundary sized particles in a classification zone, classification efficiency is 1.5-2 times higher than that in a stationary case.

This work was supported by as part of the program to improve the competitiveness of the Tomsk State University and Russian Foundation for Basic Research (project number 14-08-00367/13 NC).

\section{References}

[1] A. Roslyak, P. Zyatikov. Centrifugal air classification micropowders. Tomsk Univ. University Press, 223 (2010)

[2] A. Schwab, V. Khairullina. Investigation of the influence of unsteady swirling the turbulent flow on the motion of a single solid particle. J. Appl. 52, No. 1, 47. (2011)

[3] R.F. Patent No. 2407601 IPC B07B7/083 method centrifugal air classification of powders and device for its implementation / Zyatikov P.N., Roslyak A.T., Vasenin I.M. Schwab A.V., Demidenko A.A., garden - retdinov Sh.R. / / publ ., BI Number 36, 27.12.2010 